\title{
Focused Abdominal Computed Tomography in Clinically Suspected Adolescent Acute Appendicitis
}

\author{
Muhammad Imran Aslam ${ }^{\text {a }}$, Muhammad Osman Karim ${ }^{\text {b, d, }}$ \\ Syed Hussain Abbas ${ }^{\mathrm{b}}$, Ashifa Khan ${ }^{\mathrm{c}}$
}

\begin{abstract}
Background: Acute appendicitis (AA) is one of the most common causes of acute non-traumatic abdominal pain. The use of computed tomography (CT) in facilitating the diagnosis of AA in patients above the age of 50 where right iliac fossa (RIF) pain may represent a wide spectrum of differentials is well established. However, few studies have explored the value of low radiation, unenhanced focused abdominal computed tomography (FACT) in adolescent patients where AA represents the main differential for RIF pain. In this study, we aimed to examine the diagnostic accuracy of FACT scan in diagnosing AA in adolescent patients at a tertiary teaching children's hospital.
\end{abstract}

Methods: The study was based on cross-sectional design and occurred over a 6-month period between November 2008 and May 2009. A prospective cohort of 95 patients between the ages of $10-$ 21 years with clinically suspected AA satisfied the inclusion criteria. Patients with recent abdominal surgery or those who were pregnant were excluded from the study. The necessary institutional ethical approval was granted prior to study commencement.

Results: There was slight male preponderance of 51 patients $(54 \%)$ and 44 females $(46 \%)$. The mean age of AA was $12.75 \pm 2.7$ years (range 10 - 21). Statistical analysis showed FACT scan was $97.32 \%$ sensitive, $88.42 \%$ specific, with a positive predictive value of $98.8 \%$ and a negative predictive value of $80.0 \%$ in diagnosis of AA. The overall diagnostic accuracy of FACT scan in our study was $96.8 \%$.

Conclusions: Unenhanced FACT scan is rapid, cost-effective and safe in diagnosis of adolescent AA.

Manuscript submitted March 2, 2018, accepted March 20, 2018

aDepartment of General Surgery, Warwick Hospital, Lakin Road, Warwick, CV34 5BW, UK

bDepartment of General Surgery, Buckinghamshire Healthcare NHS Foundation Trust, Wycombe Hospital, High Wycombe, UK

'Department of Radiology, Children Hospital Lahore, Lahore, Pakistan

${ }^{\mathrm{d} C}$ Corresponding Author: Muhammad Osman Karim, Department of General Surgery, Buckinghamshire Healthcare NHS Foundation Trust, Wycombe Hospital, Queen Alexandra Road, High Wycombe, HP11 2TT, UK.

Email: m_osmankarim@hotmail.com

doi: https://doi.org/10.14740/jcs339w
Keywords: CT scan; Appendicitis

\section{Introduction}

Acute appendicitis (AA) is the most common general surgical emergency worldwide requiring a prompt diagnosis to prevent progression to gangrene and perforation [1]. It is associated with a low-fiber diet with a life-time risk of approximately one in seven individuals being affected [2,3]. In most instances the clinical diagnosis of AA may be straightforward with classical symptoms of anorexia, pyrexia and right iliac fossa (RIF) pain. However, atypical cases may result in either delay in treatment or unnecessary hospital admission and surgery [4]. Whilst the mortality in non-perforated AA is less than $1 \%$ it is known to rise as high as $5 \%$ in young and elderly patients with atypical presentations making perforation more likely [5].

AA has classically been a clinical diagnosis based on history, physical examination and laboratory findings [2, 6-8]. Therefore those with a typical history are candidates for surgery without radiological evaluation [9]. However, imaging is advisable in patients with atypical signs, pediatric patients, young woman and elderly patients particularly above the age of 50 in order to exclude other differential diagnosis [1013]. Consequently, computed tomography (CT) is becoming increasingly utilized in making the diagnosis of AA and is supported by literature demonstrating that those patients who undergo CT scan have lower negative appendectomy rates [14].

Contrast based abdominal CT scan has a reported a sensitivity of $95 \%$, specificity of $100 \%$, accuracy of $98 \%$, positive predictive value of $100 \%$ and a negative predictive value of 97\% [8]. However, conventional intravenous (IV) contrast based abdominal CT scans can be associated with contrast and radiation related risks. Patients above the age of 10 can be considered similar to adults with respect to abdomen and pelvic CT radiation dosing whereas patients younger than this cannot. Therefore, alternative lower risk protocols of CT scanning should be developed particularly when imaging younger patients [15]. Focused abdominal computed tomography (FACT) involves limiting the imaged field from the L2 vertebra to the symphysis pubis. This imaging modality has potential advantages over conventional CT by lowering the radiation dose 
Table 1. Frequency of FACT Scan Based Diagnosis of AA

\begin{tabular}{lll}
\hline & $\begin{array}{l}\text { Number of } \\
\text { patients }(\mathbf{N})\end{array}$ & $\begin{array}{l}\text { Percentage } \\
(\%)\end{array}$ \\
\hline Diagnosed on FACT scan & 85 & 89.4 \\
Not diagnosed on FACT scan & 10 & 10.5 \\
Total & 95 & 100 \\
\hline
\end{tabular}

delivered to the patient [15]. It is readily available, operator independent, relatively easy to perform, and provides results that are easy to interpret [16-20]. Furthermore, as IV contrast is not required, it is advantageous for patients with poor renal function or difficult IV access [16-20]. However at present, the use of FACT scan has not been widely adopted. Therefore, the aim of this study was to 1) determine the diagnostic accuracy of FACT scan in adolescent cases of suspected cases of AA at a tertiary teaching children's hospital; and to 2) establish if this imaging modality should be more routinely utilized.

\section{Methods}

The study was based on a cross-sectional design and occurred over a 6-month period between November 2008 and May 2009 at the Diagnostic Radiology Department located in The Children's Hospital and Institute of Child Health, Lahore, Pakistan. All patients aged between 10 - 25 years with a clinical suspicion of AA based on history and examination findings of RIF pain, nausea, vomiting and pyrexia were included in the study. Patients with previous abdominal or pelvic surgery and pregnant patients were excluded from the study. Informed consent was taken from all patients and the necessary institutional ethical approval was granted prior to study commencement.

All patients included in the study underwent FACT scan were subjected to a FACT Scan using SIEMIENS multi-slice CT, Volume zoom. Axial images were obtained from $\mathrm{L}_{2}$ vertebra level to symphysis pubis with a slice thickness of $5 \mathrm{~mm}$ and pith of 1.5. All scans were obtained without oral, rectal and IV contrast.

FACT scan findings suggestive of acute appendicitis were defined as presence of at least one of the following parameters: 1) outer to outer diameter $>6 \mathrm{~mm} ; 2$ ) appendicolith; 3) peri-appendiceal free fluid; 4) peri-appendiceal fat stranding; 5) phlegmon; and 6) abscess formation. Those patients who satisfied the criteria underwent operative intervention. Additionally, intra-operative findings were used as the ultimate gold standard of diagnosis whereby FACT scan findings were confirmed or refuted intra-operatively. All information was recorded on a study specific proforma for purposes of data collection.

Statistical analysis was performed using SPSS version 10.0. Frequency and percentage was computed for categorical variables such as gender. Mean and standard deviation was computed for quantitative variables such as age. Sensitivity, specificity, negative predictive value, positive predictive value and diagnostic accuracy of FACT scan were subsequently cal-
Table 2. Frequency of Diagnosis of AA After Surgery

\begin{tabular}{lll}
\hline & Number of patients $(\mathbf{N})$ & Percentage (\%) \\
\hline Yes & 88 & 92.6 \\
No & 7 & 7.4 \\
Total & 95 & 100 \\
\hline
\end{tabular}

culated using SPSS software.

\section{Results}

A total of 95 patients were included in the study that satisfied the inclusion and exclusion criteria. Fifty-one patients $(53.7 \%)$ were males and 44 patients $(46.3 \%)$ were females. The mean age of diagnosis of AA was $12.75 \pm 2.7$ years (range10 - 21 years). All 95 patients included were subjected to FACT scan within $2-4 \mathrm{~h}$ of presentation and underwent operative intervention. The most common finding on FACT scan was periappendiceal fat stranding demonstrable in 62 patients $(65.3 \%)$. Other common findings were outer to outer diameter more than $6 \mathrm{~mm}$ in 30 patients $(31.6 \%)$, presence of appendicolith in 20 patients $(21.1 \%)$ and peri-appendiceal fluid in 11 patients $(11.6 \%)$. Less frequently encountered findings on FACT scan were presence of phlegmon formation in four patients $(4.2 \%)$ and abscess formation in two patients $(2.1 \%)$.

Amongst the patients included in the study, 85 patients $(89.4 \%)$ had FACT scan findings suggestive of AA and 10 (10.5\%) did not meet FACT scan criteria of AA (Table 1).

The most common intra-operative finding was a thickened inflamed appendix wall found in 87 patients $(91.6 \%)$. Other common findings included the presence of peri-appendiceal fluid in 57 patients $(60 \%)$, peri-appendiceal fat stranding in 68 patients $(71.6 \%)$ and appendicolith found in 20 patients (21.1\%). Less frequently encountered inter-operative findings were phlegmon formation apparent in seven patients $(7.4 \%)$ and abscess formation apparent in four patients (4.2\%).

Eighty-eight patients (92.6\%) were confirmed to have AA after surgical intervention, whilst seven patients (7.4\%) did not demonstrate pathological findings of AA either on FACT scan or on intra-operative macroscopic findings (Table 2). However, on histological microscopic analysis of the retrieved specimen histology, it was suggestive of mild to moderate inflammation likely secondary to operative instrumentation.

All patients who were suspected of having AA on premises of clinically findings and FACT scan underwent surgery under general anesthesia (GA). Amongst the 85 patients $(89.4 \%)$ who were found to have FACT scan findings suggestive of AA, 84 patients $(88.4 \%)$ were confirmed to have AA intra-operatively. Additionally, of the 10 patients (10.5\%) who did not demonstrate the radiological findings of AA on FACT scan, two patients $(2.1 \%)$ were confirmed to have AA intraoperatively.

An appendicolith was demonstrated on FACT scan in 20 patients $(20.1 \%)$ and surgical intervention confirmed the finding of an appendicolith in the lumen of appendix (Fig. 1).

Peri-appendiceal fat stranding was the second most sig- 


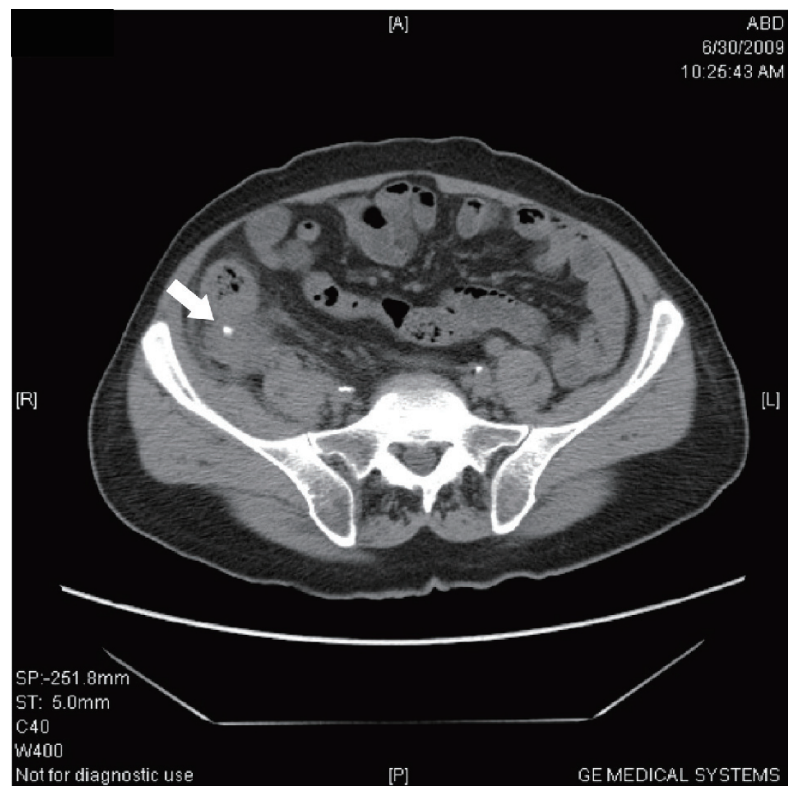

Figure 1. FACT scan demonstrating appendicolith.

nificant finding on FACT scan and was demonstrated in 62 patients $(65.3 \%)$ (Fig. 2).This finding was also demonstrated intra-operatively in 68 patients $(71.6 \%)$ therefore suggesting it to be a reliable finding on FACT scan towards radiological diagnosis of AA.

The outer to outer diameter of appendix on FACT scan of greater than $6 \mathrm{~mm}$ was demonstrated in 30 patients $(31.6 \%)$. An intra-operative inflamed thickened appendix wall was seen in 87 patients (91.6\%) (Fig. 3).

Overall, the study demonstrated that that in suspected AA, a non-enhanced FACT scan is $97.32 \%$ sensitive, $88.42 \%$ specific, $96.8 \%$ accurate with a positive predictive value of $98.8 \%$

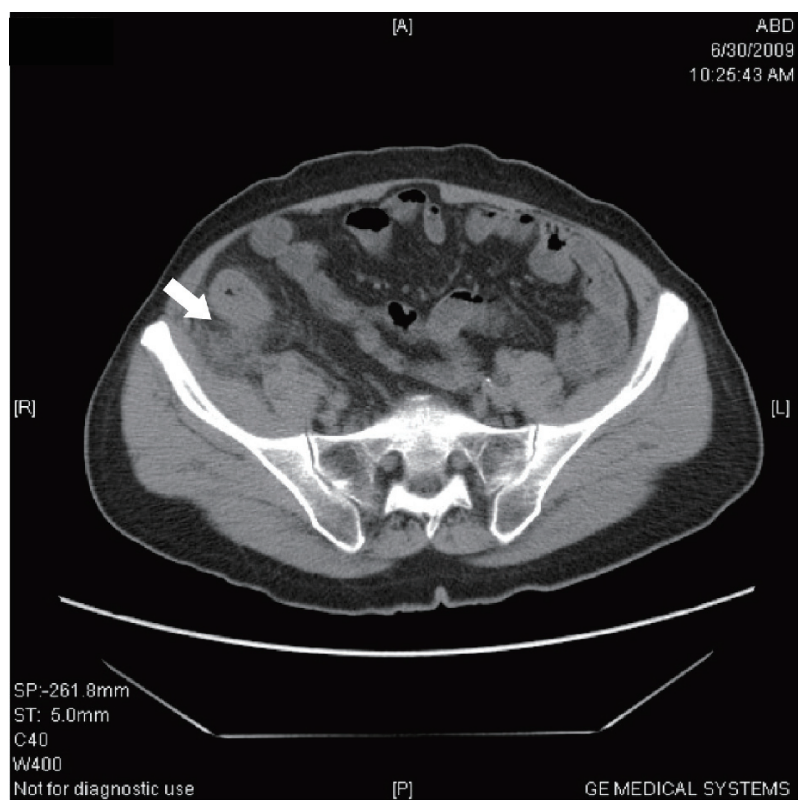

Figure 2. Peri-appendiceal fat stranding.

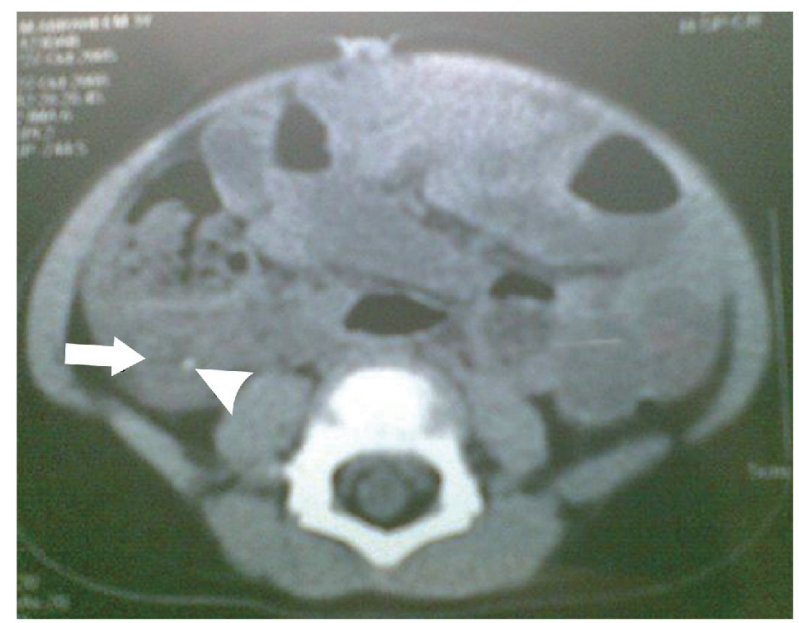

Figure 3. Axial FACT scan at level of cecum shows a thickened, tubular structure arising from cecum; this is the inflamed appendix (arrowhead). Inflammation in this region has caused marked stranding of the pericecal fat (arrow).

and negative predictive value of $80.0 \%$ (Table 3 ).

\section{Discussion}

AA is one of the most common surgical emergencies occurring worldwide [21]. There is no single sign, symptom or diagnostic test which absolutely confirms the diagnosis and morbidity is increased with diagnostic delay. Nonetheless, it is also important to minimize negative appendectomy rates due to associated operation related co-morbidity [15]. The CT scan especially after the advent of multi-detector scanner machines has been increasingly used for detection of diseased appendices and also particularly in those above the age of 50 years of exclude other pathologies such as malignancy [22-25]. Furthermore the use of thin-section CT scan significantly improves the diagnosis of acute appendicitis as shown by Weltman et al who compared $5 \mathrm{~mm}$ against $10 \mathrm{~mm}$, finding $5 \mathrm{~mm}$ slices to be superior in detecting peri-appendiceal inflammatory changes [26].

Importantly, literature suggests that contrast based abdominal CT scan is an accurate imaging technique in identifying AA with the sensitivity of $95 \%$, specificity of $100 \%$, and accuracy of $98 \%$ [8]. Our study demonstrates that in AA, a nonenhanced FACT scan is $97.32 \%$ sensitive, $88.42 \%$ specific and $96.8 \%$ accurate. Whilst it may be limited by specificity, the FACT scan has potential advantages over conventional CT imaging of the whole abdomen. FACT scan enables more rapid

Table 3. Statistical Analysis

\begin{tabular}{ll}
\hline Sensitivity & $97.32 \%$ \\
Specificity & $88.42 \%$ \\
Positive predictive value & $98.8 \%$ \\
Negative predictive value & $80.0 \%$ \\
Diagnostic accuracy & $96.8 \%$ \\
\hline
\end{tabular}


patient scanning with minimal radiation exposure, does not require IV contrast and therefore is also more cost-effective.

Most of the published studies in the field of FACT scan are contrast based. Studies on non-enhanced focal CT are less frequently encountered [15]. A Medline search using key words such as focused, abdominal, CT appendicitis revealed only seven results. To our knowledge, only one other prospective study has currently been published on this subject and encouraging compassions can therefore be made. Lane et al examined 300 consecutive patients prospectively by using non-enhanced thin-section focused helical CT for the detection of suspected AA. Their study demonstrates sensitivity of $96 \%$, a specificity of $99 \%$, and an accuracy of $97 \%$, which is supported by our results demonstrating a sensitivity of $95.4 \%$ and an accuracy of $96.8 \%$ [27]. There was no use of contrast (IV, oral or rectal) in this study. Therefore, intra-peritoneal fat was used as the principal intrinsic contrast medium of a non-enhanced FACTS scan. We demonstrated that many radiological CT scan signs of AA such as appendicolith, inflammatory stranding of mesenteric fat, appendiceal thickening, or fluid collections, abscess or phlegmon are demonstrable without the need for IV or bowel contrast administration.

Additionally, we advocate the need to highlight the sensitivity and specificity values of each radiological sign that is suggestive of AA, in order to enable a more accurate interpretation of the FACT scan findings. The most common finding on FACT scan was peri-appendiceal fat stranding found in 62 patients $(65.3 \%)$. All inflammatory pathologies localized to the appendiceal and cecal region can elicit inflammatory mesenteric fat stranding. When such stranding is demonstrated on CT, AA is an important differential to consider [28]. Results of a study evaluating the sensitivity and specificity of the several CT signs associated with AA showed that peri-appendiceal inflammation is the most sensitive sign, occurring in 100\% of 93 cases of AA [29]. The likely reason for this is that wall thickness is better-appreciated using IV contrast.

The second most common finding was outer to outer diameter more than $6 \mathrm{~mm}$ seen in 30 patients $(31.6 \%)$. This is a clinically significant finding as an enlarged appendix of greater than $6 \mathrm{~mm}$ is currently thought to be $93 \%$ sensitive and $100 \%$ specific for acute appendicitis as demonstrated in comparable studies [29]. Therefore it is important that if the appendix is found to be enlarged, a careful evaluation for peri-appendiceal inflammation is necessary to ensure the appropriate diagnosis is made.

Other important findings in our study were the presence of an appendicolith and peri-appendiceal fluid found in 20 $(21.1 \%)$ and 11 patients $(11.6 \%)$ respectively. The visualization of an appendicolith may aid in identification of the appendix on CT imaging. Lowe LH et al demonstrated an appendicolith detected on CT has a sensitivity of $65 \%$ and a specificity of $86 \%$ for diagnosing AA and has a positive predictive value of $74 \%$ and a negative predictive value of $26 \%$ [30]. Furthermore, the use of bone window settings is helpful for detecting appendicolith when evaluating patients with AA, particularly in patients where evidence of AA is equivocal [29].

The most fundamental finding of our study was to find an appendicolith intra-operatively in all patients who demonstrated this on FACT scan. This suggests that use of the FACT scan is highly accurate in diagnosing AA occurring secondary to an appendicolith. However, other studies have concluded that although an appendicolith is significantly associated with AA, the detection of an isolated appendicolith on CT is not sufficiently specific to be the sole basis for the diagnosis of AA [30]. Therefore, it is important to use other radiological criteria that have been discussed to support the radiological diagnosis of AA.

Other important considerations include that the FACT scan is faster and is available at potentially lower cost than a routine contrast-enhanced CT scan of the abdomen and pelvis. This is attributed to the fact that there is no extra preparation required or need for contrast administration. It also reduces the patient radiation burden as a limited area is scanned and a repeat scan after contrast administration is avoided.

Overall, there is limited consensus on using focused versus non-focused technique in diagnosis of AA. The principle objection to focused CT over non-focused CT could be the difficulty in identifying the cecum on the scans obtained in some patients and the failure to demonstrate other intra-abdominal pathologic conditions outside the scanning field [10]. However, given findings of this study it appears that the benefits previously described and high diagnostic yield demonstrated suggest that the FACT scan is a potentially advantageous imaging modality. This is particularly important when AA is the principal differential in adolescent patients who are less likely to have other co-existing pathologies.

It is pertinent to note the study by Rao et al which concludes that a CT scan should be used routinely in all suspected AA patients due to the very high sensitivity ( $>95 \%)$ and specificity $(>95 \%)$ for diagnosing AA [31]. This could theoretically result in a decrease in negative appendectomy rate from of 15 $20 \%$ to $<5 \%$ [32]. However, further studies would be required to establish the population risk and benefits. Additionally, the use of unnecessary radiation should be avoided and FACT scan can limit the radiation dose particularly in adolescent patients. This was an important consideration in our study as the mean age of patients in this study was $12.75 \pm 2.7$ years (range 10 21 years) with a male to female ratio of $1.2: 1$. These findings were consistent with those of previous studies that suggested a slight male predominance, with the majority of cases occurring between 13 and 25 years of age [33]. The study is limited by its application to older patient cohorts where excluded other differentials such as malignancy are particularly important.

\section{Conclusions}

With the ever increasing utilization of CT, our study favors the use of early FACT scan whenever there is suspicion of AA with equivocal findings to prevent unnecessary delays in treatment and prevent progression of AA to perforation and gangrene. The use of non-enhanced FACT scan is associated with lower costs, rapid access to imaging, a reduction in radiation exposure and hazards associated with using contrast medium. Overall, consideration of this imaging protocol can help reduce negative appendectomy rates, reduce the burden on hospital resources and optimize patient safety. Therefore, a more widespread use is advocated. 


\section{Conflict of Interest}

The authors declare that they have no conflict of interest.

\section{Funding Support}

None.

\section{Disclosure}

The authors have nothing to disclose.

\section{References}

1. Paulson EK, Kalady MF, Pappas TN. Clinical practice. Suspected appendicitis. N Engl J Med. 2003;348(3):236242.

2. Birnbaum BA, Wilson SR. Appendicitis at the millennium. Radiology. 2000;215(2):337-348.

3. Khan I, ur Rehman A. Application of alvarado scoring system in diagnosis of acute appendicitis. J Ayub Med Coll Abbottabad. 2005;17(3):41-44.

4. Old JL, Dusing RW, Yap W, Dirks J. Imaging for suspected appendicitis. Am Fam Physician. 2005;71(1):71-78.

5. Field S, Morrison I. The acute abdomen. In: Sutton D Editor, text book of radiology and imaging: 7th ed. Edinburgh; Elsevier Science Limited; 1998: 663-690.

6. Wagner JM, McKinney WP, Carpenter JL. Does this patient have appendicitis? JAMA. 1996;276(19):15891594.

7. Hallan S, Asberg A. The accuracy of C-reactive protein in diagnosing acute appendicitis - a meta-analysis. Scand J Clin Lab Invest. 1997;57(5):373-380.

8. Kessler N, Cyteval C, Gallix B, Lesnik A, Blayac PM, Pujol J, Bruel JM, et al. Appendicitis: evaluation of sensitivity, specificity, and predictive values of US, Doppler US, and laboratory findings. Radiology. 2004;230(2):472478.

9. Abou-Nukta F, Bakhos C, Arroyo K, Koo Y, Martin J, Reinhold R, Ciardiello K. Effects of delaying appendectomy for acute appendicitis for 12 to 24 hours. Arch Surg. 2006;141(5):504-506; discussioin 506-507.

10. Ashraf K, Ashraf O, Bari V, Rafique MZ, Usman MU, Chisti I. Role of focused appendiceal computed tomography in clinically equivocal acute appendicitis. J Pak Med Assoc. 2006;56(5):200-203.

11. Rosengren $\mathrm{D}$, Brown $\mathrm{AF}, \mathrm{Chu} \mathrm{K}$. Radiological imaging to improve the emergency department diagnosis of acute appendicitis. Emerg Med Australas. 2004;16(5-6):410-416.

12. Sivit CJ, Siegel MJ, Applegate KE, Newman KD. When appendicitis is suspected in children. Radiographics. 2001;21(1):247-262; questionnaire 288-294 .

13. Sivit CJ, Applegate KE, Berlin SC, Myers MT, Stallion A, Dudgeon DL, Borisa VJ, et al. Evaluation of suspected appendicitis in children and young adults: helical CT. Ra- diology. 2000;216(2):430-433.

14. Harswick C, Uyenishi AA, Kordick MF, Chan SB. Clinical guidelines, computed tomography scan, and negative appendectomies: a case series. Am J Emerg Med. 2006;24(1):68-72.

15. Pakaneh MA, Fazeli MS, Ghanaati H, Kaviani A, Aminian A, Zoberi T, et al. Non-contrast spiral computed tomography in diagnosis of acute appendicitis. Iran J Radiol. 2008;05:01-06.

16. Chang YT, Lin JY, Huang YS. Appendicitis in children younger than 3 years of age: an 18-year experience. Kaohsiung J Med Sci. 2006;22(9):432-436.

17. Albiston E. The role of radiological imaging in the diagnosis of acute appendicitis. Can J Gastroenterol. 2002;16(7):451-463.

18. Kaiser S, Frenckner B, Jorulf HK. Suspected appendicitis in children: US and CT - a prospective randomized study. Radiology. 2002;223(3):633-638.

19. Wilson EB, Cole JC, Nipper ML, Cooney DR, Smith RW. Computed tomography and ultrasonography in the diagnosis of appendicitis: when are they indicated? Arch Surg. 2001;136(6):670-675.

20. Terasawa T, Blackmore CC, Bent S, Kohlwes RJ. Systematic review: computed tomography and ultrasonography to detect acute appendicitis in adults and adolescents. Ann Intern Med. 2004;141(7):537-546.

21. Bhutta IA, Nawaz F, Mustafa J, Mudassir N. The role of high resolution ultrasonography in the diagnosis of acute appendicitis. J Rawal Med Coll. 2004;08:87-89.

22. Pittman-Waller VA, Myers JG, Stewart RM, Dent DL, Page CP, Gray GA, Pruitt BA, Jr., et al. Appendicitis: why so complicated? Analysis of 5755 consecutive appendectomies. Am Surg. 2000;66(6):548-554.

23. Poh AC, Lin M, Teh HS, Tan AG. The role of computed tomography in clinically-suspected but equivocal acute appendicitis. Singapore Med J. 2004;45(8):379-384.

24. Pickuth D, Spielmann RP. Unenhanced spiral CT for evaluating acute appendicitis in daily routine. A prospective study. Hepatogastroenterology. 2001;48(37):140142.

25. Yuksekkaya R, Akgul E, Inal M, Binokay F, Celiktas M, Aksungur E. [Unenhanced spiral CT in the diagnosis of acute appendicitis]. Tani Girisim Radyol. 2004;10(2):131139.

26. Weltman DI, Yu J, Krumenacker J, Jr., Huang S, Moh P. Diagnosis of acute appendicitis: comparison of 5and $10-\mathrm{mm} \mathrm{CT}$ sections in the same patient. Radiology. 2000;216(1):172-177.

27. Lane MJ, Liu DM, Huynh MD, Jeffrey RB, Jr., Mindelzun RE, Katz DS. Suspected acute appendicitis: nonenhanced helical CT in 300 consecutive patients. Radiology. 1999;213(2):341-346.

28. Curtin KR, Fitzgerald SW, Nemcek AA, Jr., Hoff FL, Vogelzang RL. CT diagnosis of acute appendicitis: imaging findings. AJR Am J Roentgenol. 1995;164(4):905-909.

29. Rao PM, Rhea JT, Novelline RA. Sensitivity and specificity of the individual CT signs of appendicitis: experience with 200 helical appendiceal CT examinations. J Comput Assist Tomogr. 1997;21(5):686-692. 
30. Lowe LH, Penney MW, Scheker LE, Perez R, Jr., Stein SM, Heller RM, Shyr Y, et al. Appendicolith revealed on CT in children with suspected appendicitis: how specific is it in the diagnosis of appendicitis? AJR Am J Roentgenol. 2000;175(4):981-984.

31. Rao PM, Rhea JT, Novelline RA, McCabe CJ, Lawrason JN, Berger DL, Sacknoff R. Helical CT technique for the diagnosis of appendicitis: prospective evaluation of a focused appendix CT examination. Radiology.
1997;202(1):139-144.

32. Balthazar EJ, Rofsky NM, Zucker R. Appendicitis: the impact of computed tomography imaging on negative appendectomy and perforation rates. Am J Gastroenterol. 1998;93(5):768-771.

33. Kamran H, Naveed D, Nazir A, Hameed M, Ahmed M, Khan U. Role of total leukocyte count in diagnosis of acute appendicitis. J Ayub Med Coll Abbottabad. 2008;20(3):70-71. 